\title{
PERFORMANCES AGRONOMIQUES DE LA TOMATE (Solanum lycopersicum L.) POUR SA RESISTANCE A LA MALADIE DE L'ENROULEMENT JAUNISSANT DES FEUILLES AU CENTRE DE LA CÔTE D'IVOIRE
}

\author{
J. C. NZI' ${ }^{1}$, C. KOUAME², L. FONDIO'1, S.P. A. N'GUETTA ${ }^{3}$, H. A. DJIDJI', N. K. KOUASSI ${ }^{4}$ et A. SANGARE $^{4}$
}

1Programme Cultures Maraîchères et Protéagineuses. Station de Recherche d'Anguédédou/Centre National de Recherche Agronomique. 01 BP 1740 Abidjan 01. E-mail: jcnzi2@yahoo.fr

${ }^{2}$ AVRDC-The World Vegetable Center, P.O. Box 16317 Yaoundé, Cameroon.

3UFR Bioscience. Laboratoire de Génétique. Université de Cocody. 22 BP 582 Abidjan 22.

${ }^{4}$ Laboratoire Central de Biotechnologies. Centre National de Recherche Agronomique. 01 BP 1740 Abidjan 01.

\begin{abstract}
RESUME
Cinq variétés de tomate, dont un témoin local, ont été évaluées pour leur résistance à la maladie de l'enroulement foliaire, sans traitements phytosanitaires, à la Station de Recherche sur les Cultures Vivrières du Centre National de Recherche Agronomique (CNRA) de Bouaké. Deux variétés résistantes au Tomato yellow leaf curl (CLN2126B et CLN2123A), provenaient de l'AVRDC-The World Vegetable Center et les deux autres (IDSA108, IDSA109) du CNRA. Les résultats montrent que toutes les variétés testées ont présenté des symptômes caractéristiques de la maladie. L'indice moyen de sévérité de la maladie, calculé à partir d'une échelle d'annotation, a été supérieur à 2,6 pour toutes les variétés, démontrant, ainsi, leur sensibilité. Sur l'ensemble des semis, les rendements moyens des variétés ont varié entre 1680 et $6110 \mathrm{~kg} \mathrm{ha}^{-1}$ pour CLN2116B et le témoin, respectivement. De même, les poids moyens des fruits ont varié entre 17 et $39 \mathrm{~g}$ pour CLN2123A et le témoin, respectivement. Le nombre de fruits récoltés a varié, en moyenne, entre 27 et 68 , pour CLN2116B et le témoin, respectivement. Le témoin local a ainsi été l'objet d'un regain d'intérêt grâce à ses performances, qui sont supérieures à celles des 4 autres variétés. Le témoin pourrait donc être préconisé dans des études ultérieures d'amélioration et de sélection de variétés tolérantes en culture de tomate.
\end{abstract}

Mots clés : Tomate, maladie de l'enroulement foliaire, jaunissement de la tomate, indice de sévérité, résistance.

\author{
ABSTRACT \\ AGRONOMIC PERFORMANCIES OF TOMATO (Solanum Iycopersicum L.) FOR ITS RESISTANCE TO THE TOMATO \\ YELLOW LEAF CURL DISEASE IN THE CENTRE OF CÔTE D'IVOIRE
}

Five tomato varieties, including a local check, were assessed for their resistance to Tomato yellow leaf curl disease, without phyto sanitary treatments, at the Food Crops Research Station of the National Agronomic Research Centre (CNRA) in Bouaké. Two resistant varieties to the disease (CLN2126B and CLN2123A) were obtained from AVRDC-The World Vegetable Center and the two others (IDSA108 and IDSA109) from CNRA. Results showed that all the assessed varieties presented symptoms characteristic of leaf curl. Mean severity index of this disease, calculated from a given scale of annotation, was greater than 2,6 for all varieties, demonstrating their susceptibilities. For all the sowing dates, mean variety yields varied from 1680 to $6110 \mathrm{~kg} \mathrm{ha} \mathrm{h}^{-1}$ for CLN2116B and the local check, respectively. Fruits weight varied from 17 to $39 \mathrm{~g}$, for CLN2123A and the local check, respectively. Fruits number harvested varied between 27 to 68 for CLN2116B and the local check, respectively. The local check variety was the centre of great interest, due to its performances, which were superior to those of the 4 other varieties. Therefore, it could be recommended in both improvement and selection studies for tomato culture.

Keywords : Tomato, Tomato yellow leaf curl disease, index of severity mean, resistance. 


\section{INTRODUCTION}

L'inadaptation des variétés de tomate à la saison chaude et pluvieuse et les problèmes de viroses apparaissant surtout en saison sèche figurent actuellement, parmi les contraintes majeures à la production de la tomate en Afrique de l'Ouest et, particulièrement, en Côte d'Ivoire (Anonyme, 2005). Ces dernières années, une recrudescence des viroses de type geminivirus dont les plus sévères sont le Tomato yellow leaf curl virus (Tylcv) et le Tomato leaf curl virus (Tlcv) a été observée (Moriones et Navas-Castillo, 2000 ; Qaryouti et al., 2003). Ainsi, le développement et l'étalement de la culture de tomate sur toute l'année dans le but d'assurer l'approvisionnement régulier de la population ivoirienne sont freinés par la forte pression de la virose Tylcv. Actuellement, la culture de la tomate a tendance à être abandonnée pour d'autres cultures plus rentables pendant la saison sèche telles que l'aubergine, le choux, le piment, la salade, etc. L'omniprésence et l'impact négatif de ces geminivirus sont devenus très inquiétants dans le monde tropical et subtropical (Hanafi, 2000 ; Pita, 2002 ; Delatte et al., 2003 ; Rubio et al., 2003). L'enroulement foliaire ou Tylc est de plus en plus considérée comme la maladie virale la plus dévastatrice de la tomate dans cette partie du monde.

Des prospections dans les périmètres maraîchers ont signalé la présence du Tylc dans toutes les zones de production de la tomate en Côte d'Ivoire (Kouamé et al., 1993, Fondio et al., 2001). Des travaux réalisés dans les régions centre et centre-nord de la Côte d'Ivoire ont révélé des taux d'attaque allant de 5 à $100 \%$. Des tests de produits phytosanitaires effectués à Bouaké pour la lutte chimique contre les mouches blanches vecteurs de la maladie tomato yellow leaf curl se sont révélés peu efficaces (Kouamé et al., 1997).

Des variétés de tomates tolérantes ou résistantes ont été commercialisées et d'autres sont en cours de création afin de réduire les pertes de rendement dues à cette virose (Anonyme, 2000 ; Ano et al., 2004). La plupart des variétés de tomate cultivées en Côte d'Ivoire sont des introductions et sont toutes sensibles aux souches virales locales. La sélection et la vulgarisation de variétés de tomate résistantes ou tolérantes à la maladie pourraient permettre de réduire l'impact de ce virus.
Cette étude a pour but d'évaluer les performances agronomiques de quelques variétés de tomate face à la pression de la maladie de l'enroulement jaunissant des feuilles. Ces travaux vont nous permettre d'évaluer l'incidence de la maladie sur des plants des variétés de tomate au champ. II s'agit également d'estimer l'indice moyen de sévérité et l'influence de la maladie sur les caractères de production de la tomate en fonction des dates de semis pendant la saison de prévalence du Tylc.

\section{MATERIEL ET METHODES}

\section{SITE D'ETUDE}

Cette étude a été réalisée à la Station de Recherche sur les Cultures Vivrières (SRCV) du Centre National de Recherche Agronomique (CNRA) à Bouaké, ville située au Centre de la Côte d'Ivoire $\left(7^{\circ} 46\right.$ de Latitude Nord, $5^{\circ} 06$ de Longitude Ouest et à $376 \mathrm{~m}$ d'altitude).

Le climat de cette région est caractérisé par un climat de transition entre le Sud au régime à deux saisons pluvieuses et deux saisons sèches et le Nord à une saison sèche et une saison pluvieuse. Le sol de la station de recherche est de type gravillon argileux.

\section{MATERIEL VEGETAL}

L'étude a porté sur cinq variétés de tomate dont un témoin local nommé $A B B A$, deux variétés issues de la collection du CNRA (IDSA108 et IDSA109) et deux autres variétés provenant de l'AVRDC-The World Vegetable Center et tolérantes à la maladie Tylc (CLN2116B et CLN2123A).

\section{METHODES}

Les semis ont été effectués pendant la grande saison sèche en décembre 2000 et en février 2002. Quant au semis d'octobre 2001, il se situe dans la petite saison des pluies (Figure 1). Ainsi, des essais sont mis en place à trois dates de semis : 05 décembre 2000, 22 octobre 2001 et 02 février 2002. Pour chaque essai, une pépinière est réalisée sur des planches aménagées à cet effet. Vingt et un jours après le semis, les plantes ont été repiquées sur billon. Chaque billon constitue une parcelle élémentaire 
qui comprend 24 plants disposés en deux lignes de 12 plants avec $50 \mathrm{~cm}$ entre les lignes et 40 $\mathrm{cm}$ entre plants sur la ligne. Le dispositif expérimental est un bloc de Fisher à 4 répétitions. La parcelle élémentaire est de $5 \mathrm{x}$ $2 \mathrm{~m}$ avec comme parcelle utile $5 \mathrm{~m}^{2}$.

Un épandage de fumure de fond (NPK 10 - 18 18) à raison de $300 \mathrm{~kg} / \mathrm{ha}$ a été réalisé au moment du billonnage. Deux applications d'urée $(100 \mathrm{~kg} / \mathrm{ha})$ et de potasse $(200 \mathrm{~kg} / \mathrm{ha})$ ont été effectuées à la deuxième et cinquième semaine après le repiquage. Une irrigation régulière et des sarclages manuels ont été faits à la demande. Aucun traitement phytosanitaire n'a été effectué. La récolte a été faite de manière hebdomadaire.

Les observations et mesures ont porté sur l'indice moyen de sévérité (Ims) des symptômes de la maladie qui a été quantitativement estimé au début de la floraison ( 45 jours après semis) et à maturité de la fructification qui correspond à 75 jours après semis (jas), dans chaque parcelle, de façon visuelle en utilisant une échelle de notation allant de 0 à 5 . Cette échelle de notation utilisée par loannou (1985), Kasrawi (1989) et Qaryouti et al. (2003) se présente ainsi :

$0=$ pas de symptômes visibles ;
1 = légers symptômes sur les feuilles du sommet des jeunes plants infestés ;

2 = symptômes tenaces sur les feuilles du sommet (chlorose, durcissement) pas d'effet apparent sur la croissance des plants ;

3 = syndrome yellow leaf curl incluant une faible réduction de la croissance des plants ;

4 = syndrome yellow leaf curl typique incluant une réduction sensible de la croissance des plants ;

$5=$ syndrome yellow leaf curl typique incluant une réduction évidente de la croissance des plants.

Dix plants infestés par parcelle de chaque variété ont été individuellement notés et l'indice moyen de sévérité des symptômes manifestés par la variété a été calculé par la moyenne de tous les indices. Ainsi, les variétés ayant un indice moyen de sévérité (Ims) compris entre 0 et 1,0 sont dites résistantes. Celles ayant un Ims compris entre 1,1 et 2,5 sont désignées tolérantes quand les variétés ayant un Ims compris entre 2,6 et 5 sont dites sensibles (Ioannou, 1985).

Les mesures ont aussi porté sur le nombre et le poids des fruits récoltés par parcelle pour chaque variété. Le rendement en fruits des variétés a été déterminé.

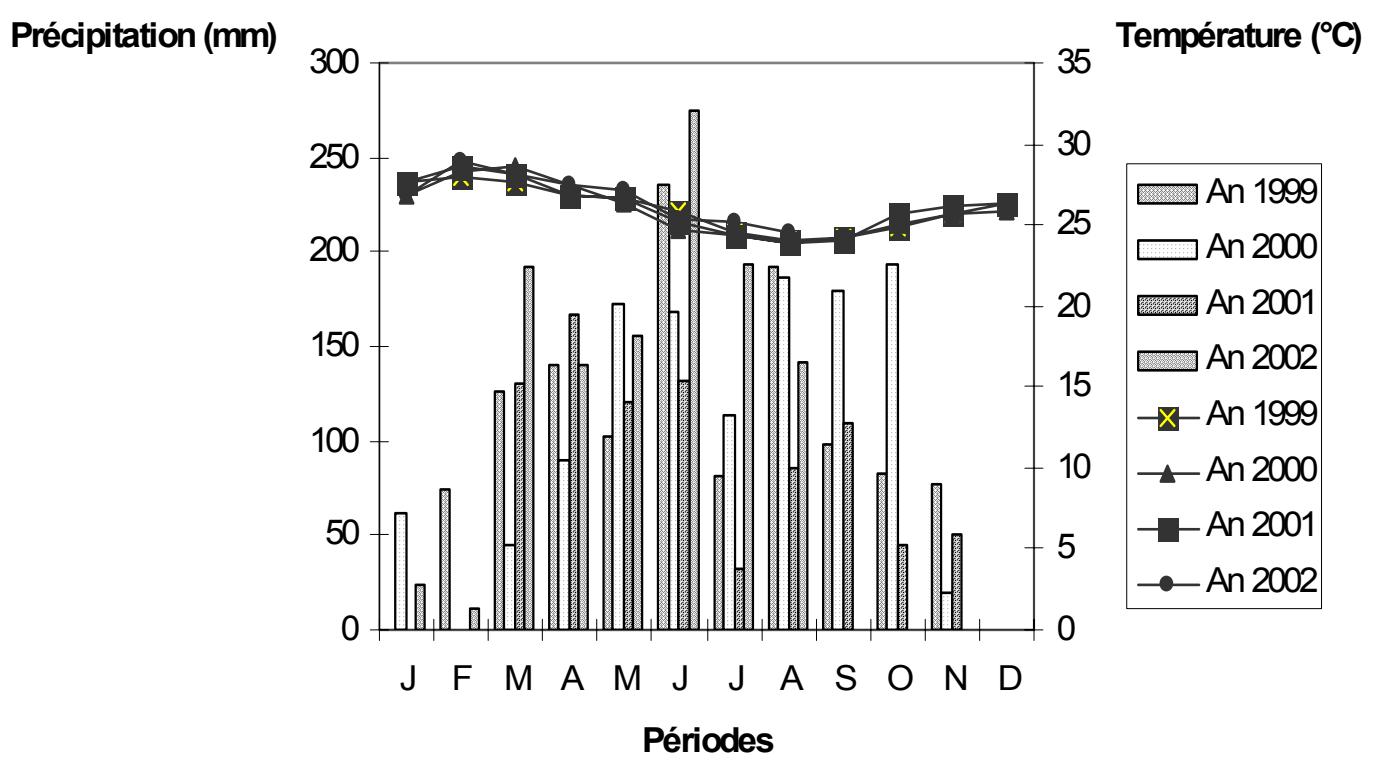

Figure 1 : Niveaux des précipitations et températures dans la zone d'étude Precipitations and temperatures levels in the zone of survey 


\section{ANALYSE STATISTIQUE}

Les données mesurées (indice de sévérité, nombre de fruits, poids moyens et rendement des fruits) ont été analysées suivant le modèle linéaire généralisé du logiciel SAS. Le test de séparation des moyennes a été effectué selon Duncan au seuil de $5 \%$.

\section{RESULTATS}

\section{INDICE MOYEN DE SEVERITE DES SYMPTÔMES DE LA MALADIE}

L'indice moyen de sévérité des symptômes des variétés de tomate a varié de 1,90 à 2,75 en octobre 2001, de 2,90 à 4,60 en février 2002 et de 3,10 à 4,60 en décembre 2000 (Tableau 1). L'analyse statistique a montré une différence significative entre les dates de semis pour cette variable. L'effet variété est significatif pour l'indice de sévérité moyen. Cependant, l'interaction variété $x$ date de semis n'a pas été significative (Tableau 2).

En décembre 2000 et février 2002, les variétés CLN2116B, IDSA108, IDSA109 et CLN2123A ont enregistré un indice moyen de sévérité supérieur à 3,80 , tandis que le témoin local ABBA en a enregistré 3,10 en décembre 2000 et 2,90 en février 2002. En octobre 2001, les indices moyens de sévérité enregistrés par toutes les variétés ont varié de 1,90 à 2,75.

\section{INDICE MOYEN DE SEVERITE ET INDICATEURS DE PRODUCTION}

\section{Rendement en fruits}

La moyenne des rendements en fruits des variétés a oscillé entre $1690 \mathrm{~kg} / \mathrm{ha}$ pour CLN2116B et $6110 \mathrm{~kg} / \mathrm{ha}$ pour le témoin local sur l'ensemble des semis (Tableau 3 ). En décembre 2000, les rendements ont varié de $570 \mathrm{~kg} / \mathrm{ha}$ avec la variété CLN2116B à $2800 \mathrm{~kg} / \mathrm{h}$ a pour le témoin local. La moyenne des rendements enregistrée est de $1210 \mathrm{~kg} / \mathrm{ha}$. Au cours du semis d'octobre 2001, les rendements des variétés ont varié de $4450 \mathrm{~kg} / \mathrm{ha}$ pour la variété CLN2116B à $13700 \mathrm{~kg} / \mathrm{ha}$ pour le témoin local. La moyenne des rendements est de $8210 \mathrm{~kg} / \mathrm{ha}$. En février 2002 , les rendements des variétés de tomate ont oscillé entre $40 \mathrm{~kg} / \mathrm{ha}$ pour CLN2116B et
$1925 \mathrm{~kg} / \mathrm{ha}$ pour le témoin local. La moyenne enregistrée est de $590 \mathrm{~kg} / \mathrm{ha}$ pour le semis de février. Les variétés IDSA108, IDSA109 et CLN2123A possèdent des valeurs intermédiaires. L'analyse de variance a montré une différence hautement significative entre les variétés et entre les dates de semis pour la variable rendement en fruits. L'interaction variété $x$ date de semis est aussi significative pour le rendement en fruits.

\section{Poids moyen des fruits}

Le poids moyen des fruits des variétés a varié de $17 \mathrm{~g}$ pour la variété CLN2123Aà $39 \mathrm{~g}$ pour le témoin local (Tableau 4).

Pour le semis de décembre 2000 , le poids moyen des fruits enregistré est compris entre $17 \mathrm{~g}$ pour la variété CLN2123A et $34 \mathrm{~g}$ pour le témoin local. Au cours du semis de février 2002, le poids moyen des fruits enregistré a évolué de $3 \mathrm{~g}$ avec la variété CLN2123A jusqu'à $30 \mathrm{~g}$ pour le témoin local. Pour le semis d'octobre 2001, le témoin local a enregistré un poids moyen des fruits de $53 \mathrm{~g}$ suivi de la variété IDSA109 avec $51 \mathrm{~g}$. La variété CLN2123A a enregistré le poids le plus bas avec $34 \mathrm{~g}$. L'analyse statistique indique une différence hautement significative entre les variétés et entre les dates de semis pour le poids moyen des fruits.

\section{Nombre moyen de fruits récoltés}

Le nombre moyen de fruits récoltés par parcelle a varié de 27 fruits pour la variété CLN2116B à 69 fruits pour le témoin local sur l'ensemble des semis (Tableau 5). La moyenne du nombre de fruits récoltés est de 28 au semis de décembre 2000, de 15 au semis de février 2002 et de 96 au semis d'octobre 2001.

Au semis de décembre 2000, le témoin local a produit 43 fruits contre 35 fruits pour la variété CLN2123A. La variété IDSA 109 a enregistré 15 fruits. Au semis de février 2002, le nombre de fruits a varié de 4 pour la variété CLN2116B à 33 fruits pour le témoin local. Le nombre de fruits est de 12 pour IDSA108 et IDSA109 quant la variété CLN2123A enregistre 11 fruits. Au semis d'octobre 2001, le témoin local en a enregistré 130 et la CLN2116B a obtenu le nombre de fruits le plus bas avec 61 fruits. Une différence statistique est observée entre les variétés de tomate et entre les dates de semis pour le nombre de fruits par parcelle. L'interaction variété $x$ date de semis est aussi significative. 
Tableau 1 : Indice de sévérité moyen des variétés de tomate par date de semis.

Severity mean index of the tomato varieties by sowing date.

\begin{tabular}{lcccc} 
& \multicolumn{4}{c}{ Date de semis } \\
\cline { 2 - 5 } Variétés & Décembre 2000 & Octobre 2001 & Février 2002 & Moyennes \\
\hline IDSA108 & $4,40 \mathrm{a} *$ & $2,75 \mathrm{a}$ & $4,40 \mathrm{a}$ & $3,85 \mathrm{a}$ \\
IDSA109 & $4,25 \mathrm{a}$ & $2,60 \mathrm{a}$ & $4,60 \mathrm{a}$ & $3,80 \mathrm{a}$ \\
CLN2116B & $4,60 \mathrm{a}$ & $2,60 \mathrm{a}$ & $3,90 \mathrm{a}$ & $3,70 \mathrm{ab}$ \\
CLN2123A & $3,90 \mathrm{ab}$ & $2,00 \mathrm{a}$ & $4,00 \mathrm{a}$ & $3,30 \mathrm{~b}$ \\
ABBA & $3,10 \mathrm{~b}$ & $1,90 \mathrm{a}$ & $2,90 \mathrm{~b}$ & $2,60 \mathrm{c}$ \\
\hline Moyennes & $4,05 \mathrm{a}$ & $2,40 \mathrm{~b}$ & $4,00 \mathrm{a}$ & 3,50 \\
Probabilité & 0,0355 & 0,2885 & 0,0062 & 0,0001 \\
C.V. & 15,04 & 28,70 & 13,67 & 16,68 \\
\hline * Les moyennes suivies de la même lettre dans la même colonne ne sont pas significativement différentes au \\
seuil de 5 \%. Means with the same letter in the same column are not significantly different at 5\%.
\end{tabular}

Tableau 2 : Valeurs des F des analyses de variance de l'indice de sévérité, du rendement, du poids moyen du fruit et du nombre de fruits par parcelle.

$F$ Values of the analysis of variance of the severity index, the fruit yield, the average of fruit weight and the fruits number.

\begin{tabular}{|c|c|c|c|c|c|}
\hline \multirow[b]{2}{*}{ Caractères } & \multicolumn{4}{|c|}{ Sources de variation } & \multirow{2}{*}{$\begin{array}{l}\text { Coefficient } \\
\text { de Variation }\end{array}$} \\
\hline & Bloc & $\begin{array}{l}\text { Date de } \\
\text { semis }\end{array}$ & Variété & $\begin{array}{c}\text { Variété } \mathrm{x} \text { Date de } \\
\text { semis }\end{array}$ & \\
\hline Indice de sévérité & $0,1^{\mathrm{ns}}$ & $53,0^{* *}$ & $9,6^{* *}$ & $0,9^{\text {ns }}$ & 16,7 \\
\hline Rendement en fruits & $4,8^{*}$ & $585,8^{* *}$ & $53,9^{* *}$ & $15,0^{* *}$ & 23,5 \\
\hline Poids moyen des fruits & $0,7^{\mathrm{ns}}$ & $66,1 * *$ & $12,4^{* *}$ & $1,1^{\mathrm{ns}}$ & 30,5 \\
\hline Nombre moyen de fruits & $4,2^{*}$ & $286,1^{* *}$ & $21,9^{* *}$ & $2,7^{*}$ & 25,4 \\
\hline
\end{tabular}

** = significatif au seuil de $1 \% ;{ }^{*}=$ significatif au seuil de $5 \% ; n s=$ non significatif. ${ }^{* *}=$ significant at $1 \% ;{ }^{*}=$ significant at of $5 \% ;$ ns $=$ not significant.

Tableau 3 : Moyennes du rendement des variétés de tomate par date de semis Averages of the tomato varieties yield by sowing date

\begin{tabular}{lcccc} 
& \multicolumn{3}{c}{ Date de semis } & \multirow{2}{*}{ Moyennes } \\
\cline { 2 - 4 } Variétés & Décembre 2000 & Octobre 2001 & Février 2002 & \\
\hline IDSA108 & $975 \mathrm{bc}^{*}$ & $7700 \mathrm{~b}$ & $590 \mathrm{~b}$ & $3088 \mathrm{~b}$ \\
IDSA109 & $640 \mathrm{bc}$ & $8460 \mathrm{a}$ & $325 \mathrm{~b}$ & $3142 \mathrm{~b}$ \\
CLN2116B & $570 \mathrm{c}$ & $4450 \mathrm{c}$ & $40 \mathrm{~b}$ & $1687 \mathrm{c}$ \\
CLN2123A & $1070 \mathrm{~b}$ & $6900 \mathrm{~b}$ & $75 \mathrm{~b}$ & $2682 \mathrm{~b}$ \\
ABBA & $2800 \mathrm{a}$ & $13700 \mathrm{a}$ & $1925 \mathrm{a}$ & $6142 \mathrm{a}$ \\
\hline Moyennes & $1211 \mathrm{~b}$ & $8242 \mathrm{a}$ & $591 \mathrm{c}$ & 3348 \\
Probabilité & 0,0001 & 0,0001 & 0,0001 & 0,0001 \\
C.V. & 23,38 & 12,17 & 63,38 & 23,47 \\
\hline
\end{tabular}

* Les moyennes suivies de la même lettre dans la même colonne ne sont pas significativement différentes au seuil de $5 \%$. Means with the same letter in the same column are not significantly different at $5 \%$. 
Tableau 4 : Poids moyens des fruits des variétés de tomate par date de semis.

Averages fruit weight of the tomato varieties by sowing date.

\begin{tabular}{lcccc}
\cline { 2 - 4 } & \multicolumn{3}{c}{ Date de semis } & \multirow{2}{*}{ Moyennes } \\
\cline { 2 - 4 } Variétés & Décembre 2000 & Octobre 2001 & Février 2002 \\
\hline IDSA108 & $19 \mathrm{bc}^{*}$ & $40 \mathrm{~b}$ & $20 \mathrm{ab}$ & $26,3 \mathrm{bc}$ \\
IDSA109 & $27 \mathrm{ab}$ & $51 \mathrm{a}$ & $12 \mathrm{bc}$ & $30 \mathrm{~b}$ \\
CLN2116B & $20 \mathrm{bc}$ & $38 \mathrm{~b}$ & $6,50 \mathrm{c}$ & $21,5 \mathrm{~cd}$ \\
CLN2123A & $17 \mathrm{c}$ & $34 \mathrm{~b}$ & $3 \mathrm{c}$ & $18 \mathrm{~d}$ \\
ABBA & $34 \mathrm{a}$ & $53 \mathrm{a}$ & $30 \mathrm{a}$ & $39 \mathrm{a}$ \\
\hline Moyennes & $23,4 \mathrm{~b}$ & $43,2 \mathrm{a}$ & $14,3 \mathrm{c}$ & 27 \\
Probabilité & 0,0061 & 0,0021 & 0,0029 & 0,0001 \\
C.V. & 25,03 & 13,87 & 57,11 & 26,50 \\
\hline
\end{tabular}

* Les moyennes suivies de la même lettre dans la même colonne ne sont pas significativement différentes au seuil de $5 \%$. Means with the same letter in the same column are not significantly different at $5 \%$.

Tableau 5 : Nombres moyen de fruits par parcelle des variétés de tomate par date de semis.

Averages number of fruits of tomato varieties by sowing date.

\begin{tabular}{lcccc}
\cline { 2 - 4 } & \multicolumn{3}{c}{ Date de semis } & \multirow{2}{*}{ Moyennes } \\
\cline { 1 - 4 } Variétés & Décembre 2000 & Octobre 2001 & Février 2002 & \\
\hline IDSA108 & $28 \mathrm{ab}^{*}$ & $99 \mathrm{a}$ & $12 \mathrm{~b}$ & $46 \mathrm{bc}$ \\
IDSA109 & $15 \mathrm{~b}$ & $84 \mathrm{~b}$ & $12 \mathrm{~b}$ & $37 \mathrm{c}$ \\
CLN2116B & $16 \mathrm{~b}$ & $61 \mathrm{c}$ & $4 \mathrm{~b}$ & $27 \mathrm{~d}$ \\
CLN2123A & $35 \mathrm{a}$ & $104 \mathrm{~b}$ & $11 \mathrm{~b}$ & $50 \mathrm{~b}$ \\
ABBA & $43 \mathrm{a}$ & $130 \mathrm{a}$ & $33 \mathrm{a}$ & $69 \mathrm{a}$ \\
\hline Moyennes & $28 \mathrm{~b}$ & $96 \mathrm{a}$ & $15 \mathrm{c}$ & 47 \\
Probabilité & 0,0064 & 0,0002 & 0,0006 & 0,0001 \\
C.V. & 36,05 & 14,22 & 47,09 & 25,37 \\
\hline * Les moyennes suivies de la même lettre dans la même colonne ne sont pas significativement différentes
\end{tabular}

au seuil de $5 \%$.

\section{DISCUSSION}

INCIDENCE DE LA VIROSE SUR LES VARIETES DE TOMATE ET INDICE MOYEN DE SEVERITE DES SYMPTÔMES

La réaction des variétés testées vis-à-vis de la maladie indique qu'aucune d'entre elles n'est résistante. En effet, elles ont toutes développé les symptômes caractéristiques de la maladie de l'enroulement jaunissant des feuilles de la tomate. Des auteurs tels que Moriones et NavasCastillo, 2000 ; Qaryouti et al., 2003 ; Rubio et al., 2003 ont eu des résultats similaires dans le monde subtropical. Au niveau tropical, Gilbertson et Shetty (2006) ont conduit des travaux en
Afrique de l'Ouest, particulièrement au Mali sur la virose. Les résultats confirment la menace que constitue le Tylcv pour la culture de la tomate partout dans le monde tropical et subtropical.

Le comportement des variétés testées n'a pas été homogène vis-à-vis de la maladie de l'enroulement jaunissant de la tomate. En effet, une variabilité des symptômes foliaires a été observée dans le champ selon les variétés et les paramètres étudiés. Ces symptômes partent du simple enroulement à l'enroulement avec jaunissement et avec des aspects du feuillage assez diversifiés suggérant ainsi, la présence probable d'au moins deux virus différents dans les champs comme l'ont confirmée les travaux de Gilberston et Shetty (2006) sur la 
caractérisation des virus responsables de l'enroulement jaunissant des feuilles de la tomate au Mali.

L'infection naturelle des parcelles a mis en évidence la sensibilité des variétés de tomate au virus. Celles-ci ont moins bien exprimé leur potentiel agronomique en présence de la maladie, hormis le témoin local.

Les effets significatifs des variétés et des dates de semis indiquent que les variétés de tomate et les dates de semis se sont exprimées différemment pour l'indice de sévérité des symptômes. Les variétés de tomate ont toutes été sensibles à divers niveaux à la maladie virale au semis de décembre 2000 et en février 2002. Alors qu'en octobre 2001, l'expression des variétés a été différente. La variété CLN2123A et le témoin local ont été tolérantes. Les rendements des variétés enregistrés dans cette période sont élevés comparés aux autres dates de semis.

Sur l'ensemble des trois semis, les moyennes des indices de sévérité des symptômes exprimés ont été semblables pour les variétés IDSA108 $(3,85)$ IDSA109 (3,80), CLN2116B $(3,70)$ et CLN2123A $(3,30)$ (Tableau 1). Ces quatre variétés ont été sensibles à la maladie. Le témoin local a enregistré un indice de 2,60. II pourrait être considéré comme une variété tolérante à la virose. En effet, durant toute l'expérimentation le témoin local a enregistré des performances meilleures comparées à celles des autres variétés tant au niveau de la contamination qu'à celui des caractères de production.

L'évolution de la maladie au cours du temps semble lente chez le témoin local, ce qui laisse présager d'un certain niveau de tolérance à la maladie Tylc chez ce cultivar. Cette tolérance pourrait s'expliquer par sa bonne rusticité qui lui permet de mieux s'adapter et de se stabiliser.

Contrairement au témoin local, les variétés CLN2116B et CLN2123A qui sont pourtant reconnues tolérantes aux virus du Tylc par I'AVRDC ont manifesté fortement les symptômes de la maladie aux différentes dates de semis. Ce résultat contradictoire pourrait s'expliquer par une différence d'agressivité des virus présents dans la zone d'étude d'une part, et d'autre part, par le vecteur Bemisia tabaci qui serait capable de transmettre de façon sélective un virus donné du complexe viral qui engendre le Tylc (Moriones et Navas-Castillo, 2000). La résistance ou la tolérance à une maladie donnée serait donc fonction de l'environnement et des interactions entre les différents facteurs pédoclimatiques. C'est pourquoi, une variété reconnue résistante dans une zone donnée peut ne plus l'être dans une autre.

Les plants de tomate issus du semis de février 2002 ont subi les dégâts les plus sévères de la maladie au champ. La variété CLN2116B a particulièrement manifesté les symptômes de jaunissement de la maladie plus que les autres variétés au semis de décembre 2000. Avec un indice moyen de sévérité des symptômes inférieur à celui enregistré lors des autres dates de semis, la période d'octobre pourrait représenter le début de l'installation de la maladie sur la station de recherche, tandis que la période de décembre à février pourrait être considérée comme la période de forte pression de la maladie.

INDICE MOYEN DE SEVERITE DES SYMPTOOMES ET INDICATEURS DE PRODUCTION

L'impact de la maladie sur les caractères agronomiques que sont le nombre de fruits récoltés, le poids moyen des fruits, et le rendement en fruits est lié généralement à la sévérité des symptômes chez certaines variétés alors que chez d'autres, il varie selon le paramètre étudié (rendement, nombre et poids moyen des fruits).

Les effets des variétés et des dates de semis sont hautement significatifs par conséquent, les variétés de tomate et les dates de semis se sont exprimées différemment pour les différents caractères agronomiques mesurés.

Les rendements du semis d'octobre 2001 ont généralement été supérieurs à ceux des trois semis confondus (Tableau 3). A cette période, l'incidence de la maladie sur le rendement des variétés a été moindre comparée à celles de décembre 2000 et février 2002. Les conditions ont donc été beaucoup plus favorables pour l'expression des variétés. Le rendement moyen de l'ensemble des semis étant de $3350 \mathrm{~kg} / \mathrm{ha}$, le semis d'octobre 2001 a donc été le plus productif vis-à-vis de la maladie de l'enroulement jaunissant des feuilles de la tomate. Le témoin local a enregistré un rendement d'environ deux fois plus élevé que ceux des autres variétés.

Avec le semis d'octobre 2001, les variétés de tomate ont enregistré des nombres de fruits 
relativement plus élevés que ceux obtenus avec les deux autres semis. Le témoin local et la variété CLN2123A malgré des niveaux de production assez réduits, ont été les plus productifs en fruits. Les fruits produits ont été généralement de petites tailles. Néanmoins, les poids moyens de ces fruits ont été supérieurs à ceux des fruits produits par les mêmes variétés lors des semis de décembre 2000 et de février 2002. Les poids moyens des fruits les plus faibles de l'étude ont été obtenus au cours du semis de février 2002. Les poids moyen des fruits des variétés étudiées ont été dans l'ensemble très faibles (Tableau 4). En effet, ceux des variétés CLN2116B (21 g) et CLN2123A $(17 \mathrm{~g})$ sont beaucoup inférieurs à leurs poids standards homologués par l'AVRDC-The World Vegetable Center qui sont de $50 \mathrm{~g}$ pour CLN2116B et $60 \mathrm{~g}$ pour CLN2123A (Anonyme, 2000). Le nombre de fruits récoltés par parcelle et le poids moyen des fruits sont deux variables qui influencent directement le rendement (N'zi et al., 2000). Ceci confirme l'impact négatif de la maladie virale sur la production de la tomate dans les pays où sévit la virose (Hanafi, 2000 ; Delatte et al., 2003 ; Qaryouti et al., 2003). De plus, plusieurs plants n'ont pu produire de fruits lorsque d'autres en ont produit très peu de tailles réduites (Delatte et al., 2003 ; Anonyme, 2005). Plus, l'infection est précoce plus grande est l'incidence de la maladie. La contamination précoce des plants au champ peut aussi empêcher la formation des fruits, ou faire avorter les fleurs et chuter les fruits déjà formés. En effet, les plants de tomate au champ au cours des différentes dates de semis ont subi très tôt la colonisation du vecteur de la maladie : la mouche blanche Bemisia tabaci. Ce qui a eu pour conséquence une réduction considérable du rendement des fruits et de ses composantes. Aussi, la période d'harmattan qui sévit à Bouaké de décembre à février a eu un impact négatif en accentuant l'impact de la maladie.

\section{CONCLUSION}

Cette étude donne une idée globale de la présence effective de la maladie chez toutes les variétés de tomate au champ. Cette maladie est essentiellement due à la mouche blanche, agent vecteur. En absence de traitements phytosanitaires l'impact de la maladie en milieu réel a été estimé, une seule larve ou un adulte de la mouche blanche est capable de transmettre le virus de la maladie à une plante saine. La sévérité des symptômes exprimés par les variétés a permis de déterminer la sensibilité de toutes les variétés de tomate à la maladie. Les valeurs des indicateurs de production de la tomate ont été toutes réduites. Cependant, le témoin local a subi une infection virale plus faible que les autres variétés. Le témoin a été plus tolérant à la maladie que les autres variétés. Celles dites tolérantes par l'AVRDC-The World Vegetable Center ont été beaucoup sensibles à la maladie. La grande variabilité des symptomes manifestés par les plants attaqués au champ suggère soit, la présence d'espèces ou de souches virales particulières en Côte d'Ivoire, soit le changement de comportement des variétés de tomate importées et cultivées.

La caractérisation de la diversité biologique et moléculaire de tous les virus responsables de l'enroulement foliaire de la tomate en Côte d'Ivoire s'avère nécessaire en vue de lutter plus efficacement contre la maladie.

\section{REFERENCES}

Ano G., Anaïs G., Marival P. et A. Chidiac. 2004. L'amélioration de la tomate pour les régions tropicales de plaines : travaux en Guadeloupe. Phytoma, La défense des végétaux $573: 23$ - 25.

Anonyme. 2000. AVRDC Tomato Breeding. Tomato inbred lines for international distribution. AVRDC, Sheet 3, Taiwan, $3 \mathrm{p}$.

Anonyme. 2005. AVRDC Tomato Breeding. Sources of Resistance to Whiteflytransmitted Geminiviruses. AVRDC, Sheet 3, Taiwan, $5 \mathrm{p}$.

Delatte H., Dalmon A., Rist D., Soustrade I., Wuster G., Lett J. M., Goldbach W. R., Peterschmitt M. and B. Reynaud. 2003. Tomato yellow leaf curl virus can be acquired and transmitted by Bemisia tabaci (Gennadius) from tomato fruit. Plant Dis. 87 (1) : 1297 - 1300.

Fondio L., Aïdara S., Djidji A. H., Zohouri G. P. et G. P. Gnonhouri. 2001. Diagnostic prophylactique du secteur maraîcher de la région d'Abidjan (Délégation Régionale Sud de I'ANADER) : Contraintes et ébauches de solutions. CNRA, Rapport d'étude, Bouaké, Côte d'Ivoire, $28 \mathrm{p}$. 
Gilberston R. et R. Shetty . 2006. Application de la biotechnologie pour lutter contre la virose de la tomate en Afrique de l'ouest. Activités de développement. Croissance économique accélérée. Projet USAID-AVRDC. http://www.usaid.gov/, 16 novembre 2006, USAID, Mali, $3 \mathrm{p}$.

Hanafi A. 2000. La mouche blanche et le virus des feuilles en cuillère de la tomate (Tylcv). Transfert de technologie en Agriculture. Bulletin mensuel d'information et de liaison du PNTTA. MADREF/DERD, Rabat, Maroc. $\mathrm{N}^{\circ} 73.4 \mathrm{p}$.

loannou N. 1985. Yield losses and resistance of tomato to strains of tomato yellow leaf curl and tobacco mosaic viruses. Agricultural Research Institute. Ministry of Agriculture and Natural Resources. Technical Bulletin 66. Cyprus : $11 \mathrm{p}$.

Kasrawi M. A. 1989. Inheritance of resistance to tomato yellow leaf curl virus (Tylcv) in Lycopersicon pimpinellifolium. Plant Dis. 73435 - 437.

Kouamé C. N., Adou A., Nguessan K. P., Kouakou A. et A. H. Djidji. 1993. Enquête diagnostique des productions maraîchères en Côte d'Ivoire. IDESSA, Bouaké, Côte d'Ivoire, $30 \mathrm{p}$.
Kouamé C. N., Djidji A. H. et L. Fondio. 1997. Rapport d'achèvement du projet de développement de la culture de la tomate d'industrie en région Centre et Centre-Nord de la Côte d'Ivoire. IDESSA, 100 p.

Moriones E. and J. Navas-Castillo. 2000. Tomato yellow leaf curl virus, an emerging virus complex causing epidemics worldwide. Vir. Resear. 71: 123 - 134.

N'zi J. C., Kouamé C., Fondio L. et A. H. Djidji. 2000. Etude de l'interaction génotype $x$ environnement sur les performances de la tomate (Lycopersicon esculentum Mill.). Agron. Afr. 12 (2) : 51 - 60.

Pita S. P. 2002. Diversité génétique des geminivirus (Begomovirus sp.) infectant le manioc (Manihot esculenta, Crantz) en Afrique. Thèse de doctorat, UFR Biosciences, Université d'Abidjan-Cocody (Côte d'Ivoire), $144 \mathrm{p}$.

Qaryouti M. M., Hurani O. M. and A. Y. Mahadeen. 2003. Susceptibility of Jordanian tomato landraces to tomato yellow leaf curl virus. Plant Gen. Res. News. 136 : 31 - 33.

Rubio L., Herrero J. R., Sarrio J., Moreno P. and J. Guerri. 2003. A new approach to evaluate relative resistance and tolerance of tomato cultivars to begomoviruses causing the tomato yellow leaf curl disease in Spain. Plant Path. 52 : 763 - 769. 